\title{
First Principles Study of Sensitizers for Dye Sensitized Solar Cells: Effects of Adsorption Modes and Anchoring Groups
}

\author{
Santhanamoorthi Nachimuthu, Wei-Chieh Chen and Jyh-Chiang Jiang* \\ National Taiwan University of Science and Technology, Department of Chemical Engineering, \\ Taipei, Taiwan (ROC) \\ jcjiang@mail.ntust.edu.tw
}

\section{Extended Abstract}

Dye-sensitized solar cells (DSSCs) have attracted considerable attention for clean energy applications due to their potential advantages of low cost and high conversion efficiency.[1] In DSSC, the sensitizer is a key component, which is responsible for capturing of sunlight and generation of electric charges. Ru-based complexes have been primarily used as dye sensitizers, which yields overall conversion efficiencies greater than 10\% under AM1.5 irradiation.[2] Besides, metalfree organic dyes have been proposed because of scarcity of Ru metals. A typical organic sensitizers consist of a donor, $\pi$ bridge and acceptor with anchoring groups binds on the surface. Even though a lot of efforts such as substitution of different donor/acceptor moieties and by means of increasing $\pi$-spacers, have been made in the designing strategies of organic dyes to enhance the efficiency of DSSCs, one can reach up to only $12 \%$. [3]. The overall DSSC performances are predominantly determined by the electronic coupling between the dye and the semiconductor. Hence, an efficient dye, apart from the previously reported spectral and energetic requirements, should have suitable functional groups to strongly bind with the semiconducting oxide surface. Therefore, the anchoring group is a very important consideration for improving DSSC device functionality. Also, previously reported that the adsorption configuration/orientation of dyes on the semiconductor surface, affects the charge injection from the donor levels of the excited dye and the acceptor levels of the semiconductor conduction band. $[4,5]$

Here, we have designed a basic D- $\pi$-A type of organic dyes, which has 4-methoxy-N-(4-methoxyphenyl)-Nphenylbenzeneamine (MPBA) as donor, thiophene (T) as $\pi$-bridge, thienopyrazine (TP) as an acceptor and $\mathrm{COOH}$ and $\mathrm{CSSH}$ as anchoring groups and investigated their adsorption and electronic properties on $\mathrm{TiO}_{2}(101)$ anatase surface using density functional theory (DFT). All the calculations in this study have been performed using the generalized gradient approximation (GGA) with the Perdew-Burke-Ernzerhof (PBE) functional exchange-correlation functional. To find the optimum dye length for further studies, we adsorbed the dyes which has different units such as only anchoring groups, acceptor with anchoring groups, $\pi$-bridge with acceptor and anchoring groups and finally donor- $\pi$-bridge- acceptor with anchoring groups on the surface. Based on the calculated adsorption energies and electronic properties of the above systems, we found that the model dye consists of only acceptor and anchoring group is sufficient to explain the interaction between the dye and surface. Further, we have designed a model dye with only TP and COOH groups to investigate their different adsorption configuration on the surface. For this we have considered monodentate ester-type (MET), bidentate chelating (BC) and bidentate bridging (BB) adsorption configurations. We also investigated the effect of substitution of cyano group $(\mathrm{CN})$ in the anchoring part on the adsorption energy and charge injection ability. The calculated adsorption energy value show that the dye adsorbed via both carboxyl and cyano groups is more stable than the other configurations due to their strong dative and hydrogen bonding with surface. Our results indicate that even though the inclusion of cyano group can enhance the thermal stability of the dyes, it decreased both electron injection ability to the surface and dye coverage on the surface. These theoretical findings offer a path forward in the design of efficient organic dyes for practical DSSC applications.

\section{References}

[1] B. O'Regan and M. Gratzel, "A low-cost, high-efficiency solar cell based on dye-sensitized colloidal TiO2 films," Nature, vol. 353, pp. 737-740, 1991.

[2] M. Gratzel, "Photoelectrochemical cells," Nature, vol. 414, pp. 338-344, 2001. 
[3] W. H. Zhu, Y. Z. Wu, S. T. Wang, W. Q. Li, X. Li, J. A. Chen, et al., "Organic D-A-pi-A Solar Cell Sensitizers with Improved Stability and Spectral Response," Adv. Funct. Mater., vol. 21, pp. 756-763, 2011.

[4] M. Pastore and F. De Angelis, "Computational modelling of $\mathrm{TiO} 2$ surfaces sensitized by organic dyes with different anchoring groups: adsorption modes, electronic structure and implication for electron injection/recombination," PCCP, vol. 14, pp. 920-928, 2012.

[5] M. Pastore and F. De Angelis, "Aggregation of Organic Dyes on TiO2 in Dye-Sensitized Solar Cells Models: An Ab Initio Investigation,” Acs Nano, vol. 4, pp. 556-562, 2010. 